\title{
DETERMINATION OF ACETONE BY SENSITIZED ROOM TEMPERATURE PHOSPHORIMETRY IN AQUEOUS SOLUTION
}

\author{
ZUWU CHEN and JINGOU XU* \\ Department of Chemistry, Xiamen University, Xiamen, Fujian, China
}

\begin{abstract}
A sensitized room temperature phosphorimetric method for the determination of acetone in solution was developed for the first time. Acetone was excited at $274 \mathrm{~nm}$ and the phosphorescence intensity emitted from biacetyl, the energy acceptor, was measured at $515 \mathrm{~nm}$. The calibration curve was linear over the concentration range of $2.0 \times$ $10^{-4}-1.5 \times 10^{-2} \mathrm{~mol} / 1(\gamma=0.9995)$ and the limit of detection was $7.9 \times 10^{-5} \mathrm{~mol} / 1$.
\end{abstract}

Key words acetone, water, room temperature phosphorescence

Acetone is an important industrial material and chemical reagent, and is becoming increasingly common as a water pollutant because of its extensive application. The conventional methods for the microdetermination of acetone in aqueous solution are spectrophotometry $[1-3]$ and liquid chromatography $[4,5]$. Although these methods have higher sensitivity, they are subjected to complexly preliminary treatment and lack of speed, and the dynamic ranges for the determination are relatively narrow. Consequently in an attempt to overcome the drawbacks of the literature methods, an investigation was made to develop a sensitized room temperature phosphorimetry (SRTP) for the determination of microamounts of acetone in aqueous solution. This approach has been proved to be simple, rapid, and sensitive.

\section{PRINCIPLES}

Sensitized room temperature phosphorimetry in liquid solutions, which was first presented early in $1981[6]$, is based on the triplet-triplet energy transfer from the analyte (energy donor) to a suitable energy acceptor, provided that the energy donor has a very low fluorescence quantum yield but a high quantum yield for the intersystem crossing from $S_{1}$ state to $T_{1}$ state and the triplet-triplet energy transfer between the donor and the acceptor has enough efficiency, and that the acceptor has a high phosphorescence quantum yield. The analyte, therefore, can be indirectly determined by measuring the phosphorescence intensity emitted from the acceptor.

So far as we know, only biacetyl and 1,4-dibromonaphthalene have been used as the acceptor in SRTP. Since 1, 4-dibromonaphthalene has a relatively high absorbance in ultraviolet region, biacetyl was selected as the acceptor in our experiments.

Acetone does not have a conjugated system, so it can not fluoresce. However, it has a high quantum yield of intersystem crossing $\left(\varphi_{i_{1} \rightarrow t_{1}} \approx 1\right)$ and a long triplet life time $\left(\approx 10^{-5} \mathrm{~s}\right)$. Besides, its triplet energy is much higher than that of biacetyl $\triangle E \approx 23 \mathrm{kcal} / \mathrm{mol})$. Acetone, therefore, can be determined by SRTP with biacetyl as an energy acceptor.

\section{EXPERIMENTAL}

Apparatus The luminescence spectra and absorption spectra were recorded on a Hitachi 650-10 S fluorescence spectrophotometer and a Beckman DU-8B UV-VIS spectrophotometer, respectively. A TOA HM-20E pH meter was used to measure the pH of solutions. A simple and effective oxygen removal system was made by ourselves.

Reagents All reagents used were of analytical reagent grade. Distilled water was demineralized in a mixed-bed ion-exchanger column and then distilled again after addition of a small quantity of alkali and potassium permanganate. A standard $(0.10 \mathrm{~mol} / 1)$ stock solution of acetone in water and a 1.0 $\times 10^{-2} \mathrm{~mol} / 1$ aqueous solution of biacetyl were prepared. Ultrapure nitrogen (purity over 
$99.999 \%$ ) was used for the purpose of oxygen removal.

Oxygen removal system Since oxygen is an effective quencher of phosphorescence, it should be removed prior to the measurement of phosphorescence. Although the removal of oxygen from a solution may be achieved either by reacting with sodium sulfite or by passing ultrapure nitrogen through the solution, sodium sulfite is not suitable in this experiment because of its quenching effect on the phosphorescence of biacetyl. A 1-cm quartz cell was used as the oxygen removal system and constructed as described in Fig. 1, allowing efficient oxygen removal and protection of the solution from re-entrance of oxygen during the period of measurement. The quartz cell was placed in the sample compartment of the fluorescence spectrophotometer during the processes of oxygen removal and measurement. The longer glass capillary of the system, which is movable up and down, was put into the solution during oxygen removal and pulled out of the solution before measurement, as described in Fig. 1a and Fig. 1b, respectively.

Procedure To a $10-\mathrm{ml}$ standard flask add $1.0 \mathrm{ml}$ of biacetyl solution and an appropriate volume of the standard acetone solution or the tested solution, containing $\leqslant 8.7 \mathrm{mg}$ acetone, make up to volume with distilled water and mix. Transfer a portion of the solution to a $1-\mathrm{cm}$ quartz cell, then remove oxygen from the solution by passing ultrapure nitrogen through the solution for $1.5 \mathrm{~min}$ (flow rate 60 $\mathrm{ml} / \mathrm{min})$, and finally measure the phosphorescence intensity of the solution $\left(\lambda_{\mathrm{ex}}=274 \mathrm{~nm}, \lambda_{\mathrm{em}}=\right.$ $515 \mathrm{~nm}$ ).

\section{RESULTS AND DISCUSSION}

Selection of excitation and measurement wavelengths The absorption spectrum of acetone and the phosphorescence emission spectra of biacetyl were shown in Fig. 2 and Fig. 3, respectively. In order to avoid the interference from the second-order Rayleigh scattering of the excitation light with the measurement of phosphorescence, $274 \mathrm{~nm}$ rather than $267 \mathrm{~nm}$, the wavelength of maximum absorption for acetone, was chosen as the excitation wavelength. The wavelength of maximum phosphorescence emission for biacetyl, $515 \mathrm{~nm}$, was used as the measurement wavelength.

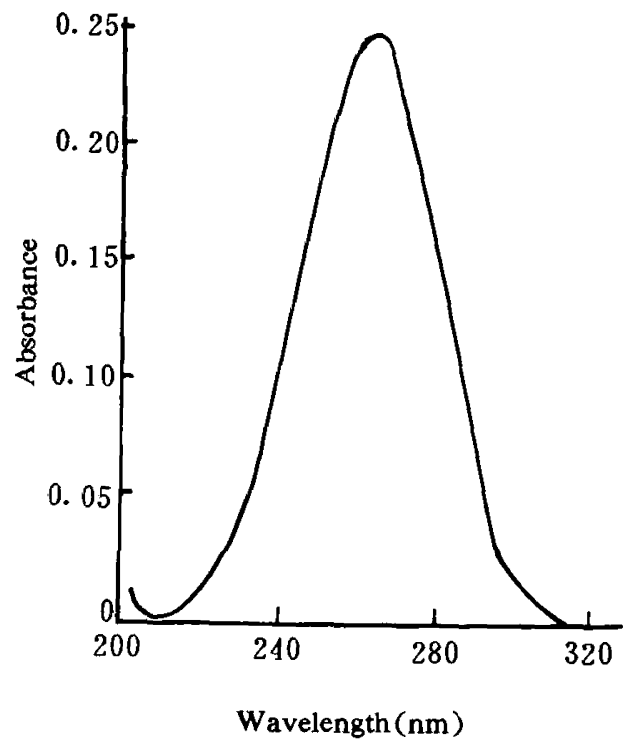

Fig. 2. Absorption spectrum of acetone

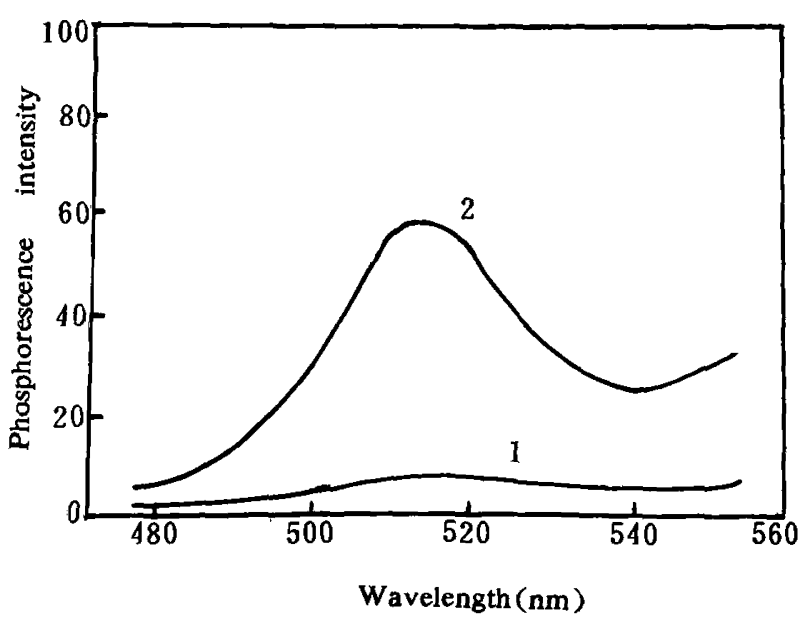

Fig. 3. Phosphorescence emission spectra of biacetyl sensitized by acetone: 1 , before oxygen removal; 2 , after oxygen removal 
Concentration of biacetyl According to literature $[6,7]$,

$$
\theta_{D A}=\frac{K_{\imath}[A]}{K_{\iota}[A]+1 / \tau_{0}^{D}}
$$

Where $\theta_{\mathrm{DA}}, \mathrm{K}_{\mathrm{t}},[\mathrm{A}]$ and $\mathrm{t}_{0}^{\mathrm{D}}$ are the efficiency of the energy transfer process, the bimolecular rate constant of transfer, the concentration of the acceptor, and the triplet lifetime of the donor in the absence of the acceptor, respectively, $50 \%$ of the donor triplets will generate the acceptor triplets if

$$
1 / \tau_{0}^{D}=K_{\imath}[A]
$$

For a diffusion-controlled reaction, $\mathrm{K}_{\mathrm{t}}$ is of the order of $10^{10} 1 \mathrm{~mole}^{-1} \mathrm{sec}^{-1}$. Substituting $\tau_{0}^{D}=$ $10^{-5} \mathrm{sec}$ (the triplet lifetime of acetone) into $E_{q}$. (2) gives $[A]=10^{-5} \mathrm{~mol} / 1$. This implies that a biacetyl concentration of $10^{-5} \mathrm{~mol} / 1$ should at least be used in order to usefully detect acetone by SRTP. As the result of tests, biacetyl concentration of $10^{-3} \mathrm{~mol} / 1$ was considered to be suitable.

Effect of $\mathrm{pH}$ The phosphorescence intensity for a fixed amount of acetone was determined by the above-mentioned procedure at various $\mathrm{pH}$ values. As a result, constant intensity was obtained at $\mathrm{pH}$ $1.0-6.0$. Therfore, a pH value of 4 was chosen for the analytical procedure and the $\mathrm{pH}$ values of solutions obtained by the analytical procedure were just about 4 .

Effect of the length of passing nitrogen through the solution When a constant flow $(60 \mathrm{ml} / \mathrm{min})$ of nitrogen was maintained, the experiment showed that the phosphorescence intensity rapidly increased as nitrogen passed through the solutions, reached the maximum at the length of $1.5 \mathrm{~min}$, and then slowly decreased with an increase of the length. The decrease of the phosphorescence intensity with an increase of the length may be due to the volatility of acetone and biacetyl.

Interference In order to assess the possible analytical applications of this new method, the effect of some foreign compounds that may often accompany acetone was examined by carrying out determinations of $120 \mathrm{ppm}$ of acetone in the presence of each of these compounds. The results are summarized in Table 1 .

The calibration curve and the detection limit of the method The results showed that the calibration curve was linear over the concentration range of $2.0 \times 10^{-4}-1.5 \times 10^{-2} \mathrm{~mol} / 1$ under the experimental conditions $(r=0.9995)$ and the limit of detection for the method was $7.9 \times 10^{-5} \mathrm{~mol} / 1$.

Table 1. Effect of foreign compounds on the determination of $(120 \mathrm{ppm})$ acetone

\begin{tabular}{lc}
\hline Interferent & $\begin{array}{c}\text { Permissible } \\
\text { concentration, ppm * }\end{array}$ \\
\hline acetic acid & $1 \times 10^{4}$ \\
ethanol & 2000 \\
ethyl acetate & 1000 \\
ethyl aldehyde & 1000 \\
ethyl ether & 4000 \\
methanol & $1 \times 10^{5}$ \\
\hline
\end{tabular}

* Amount of diverse components causing an error of less than $10 \%$ in the determination

\section{REFERENCES}

1. S. A. Rahim and W. A. Bashir, Microchem. J. , 26, 329(1981).

2. S. Falamerz, A. K. Hareez and W. A. Bashir, J. Indian Chem. Soc. , 62, 634(1985).

3. J. Ahmadzadeh and J. H. Harker, Microchem. J. , 19, 279(1974).

4. D. L. Duval, M. Rogers and J. S. Fritz, Anal. Chem. 57, 1583(1985).

5. B. A. Rudenko, N. S. Goryachev and V. V. Gavrilov, $\overline{\mathrm{Zh}}$. Anal. Khim. (Russ), 37, 544 (1982).

6. J. J. Donkerbroek, J. J. Elzas, C. Gooijer, R. W. Frei and N. H. Velthorst, Talanta, 28, 717(1981).

7. J. J. Donkerbroek, C. Gooijer, N. H. Velthorst and R. W. Frei, Anal. Chem. , 54, 891 (1982). 